\title{
SUPPLEMENTAL DATA 1
}

\section{Transgenic enhancer flies}

\section{col-TV-CRM; length: 6389 bp}

All col enhancer versions (wt and mutant) were synthesized and flanked with 5 'NotI and 3 'XbaI sites and cloned into the placZ.attB vector. All enhancer reporter construct were landed in the attP40 landing site on chromosome 2 (estimated cytolocation 25C7).

\section{col-dAp-CRM length 3475 bp:}

The col-dAp_CRM was based on the Janelia Farm Gal4 line (49248) and the enhancer fragment was generated via PCR, cloned into the TOPO vector for further sub-cloning via BamHI and NotI into the pEGFP.attB target vector. Mutated versions of the enhancer were synthesized at GenScript and delivered in a pUC57 vector. Cloning of the mutated versions was done via BamHI and NotI into the pEGFP.attB vector. Wildtype constructs were landed both on the second and third chromosome at cytolocation 28E7 (chr2) and cytolocation 68A4 (chr3). All mutated enhancer versions were landed on cytolocation 68A4.

Primer 1: CATTTTCGTGCGTCTCATCGATTCG

Primer 2: GGGACACGGGTTAACAATGTAATGC

\section{apS2-CRM; length: 1968 bp}

The apS2 enhancer fragment was generated via PCR, cloned into the TOPO vector and subcloned into the placZ.attB vector via EcoRI. Injection was done at GenetiVision and constructs were landed on second and third chromosome, in the landing sites VK31 and VK37.

Primer 1: TATCGCCGTAATCACAACAAAGT

Primer 2: CCCGCTGCCAAAACAACCCTAAG

\section{apSJ2-CRM, length: 1262 bp}

The apSJ2 WT construct was generated via PCR, cloned into the TOPO vector and subcloned into the placZ.attB vector via $3^{\prime} \mathrm{XbaI}$ and $5{ }^{`} \mathrm{BamHI} / \mathrm{BgII}$. Mutant versions were synthesized at GeneScript based on the WT construct, flanked with $3^{\prime} \mathrm{XbaI}$ and $5^{\prime} \mathrm{BamHI}$ restriction sites for site directed integration into the reporter target vector. Injection was done at BestGene and landed on chromosome 2 cytosite $28 \mathrm{E} 7$.

Primer 1: ATGTGTTTTGGTTCCAGGTCCG

Primer 2: CCCACTCATAAGCCTAGAGACA

\section{eya-CRM; length: 1307 bp}

The eya enhancer fragment was created by PCR, 5 'XhoI and 3 ' XbaI restriction sites were introduced via the primers. Fragments were cloned into the TOPO vector and subsequently cloned into the $\mathrm{pEGFP}$.attB target vector. Mutated version of the enhancer fragment were synthesized at Genescript, delivered in a pUC57 vector and cloned into the pEGFP.attB vector via XhoI and XbaI. Injection was done at BestGene. Wildtype constructs were landed both on the second and third chromosome at cytolocation 28E7 (chr2) and cytolocation 68A4 (chr3). All mutated enhancer versions were landed on cytolocation 68A4.

Primer1: XhoI; CTCGAGGATCACAGAATCCAGCT

Primer2: XbaI; TCTAGACTCGCCCAATCTCAACTC

\section{dimm-CRM; length 1224 bp}

The Dimm enhancer was generated via PCR, cloned into the TOPO vector and subsequently cloned into the $\mathrm{pEGFP}$.attB target vector via $5{ }^{`}$ BamHI and $3{ }^{\prime}$ NotI. Mutant versions of the 
enhancer were synthesized at GenScript, delivered in a pUC57 vector and cloned via BamHi and NotI into the pEGFP.attB target vector. Flies were injected at BestGene and wild type constructs were landed on both the second and third chromosome at cytolocation 28E7 (chr2) and cytolocation 68A4 (chr3). All mutated enhancer versions were landed on cytolocation 68A4.

Primer1: GTGCCAGAGCAACGCGAATTTAAG

Primer1: CTAGTGTCTTTGATTTGATGCCTTCC

\section{Nplp1-CRM; length 1586 bp}

The Nplp1 enhancer was generated via PCR, cloned into the TOPO vector and subsequently cloned into the pEGFP.attB target vector. Since the Nplp1-CRM fragment contains a promoter for Nplp1 (first exon), the fragment was inserted into the EGFP vector in reversed orientation. Cloning was done via $5^{`} \mathrm{BamHI}$ and $3{ }^{\prime}$ NotI. Mutant versions of the enhancer were synthesized at GenScript delivered in a pUC57 vector and subsequently cloned into the pEGFP.attB vector. Flies were injected at BestGene and wild type constructs were landed on both the second and third chromosome at cytolocation $28 \mathrm{E} 7(\mathrm{chr} 2)$ and cytolocation $68 \mathrm{~A} 4$ (chr3). All mutated enhancer versions were landed on cytolocation 68A4.

Primer1: CTGCCCTTTTGCAGCACCCACTTCC

Primer2: GCATGGATTGGTTGAAGCAAAAGCC 